ASCA

\section{ASCA: genetic marker, predictor of disease, or marker of a response to an environmental antigen?}

\section{F Seibold}

\section{Anti-Saccharomyces cerevisiae antibodies (ASCA) may be a marker of an immune response to an environmental antigen that occurs in the context of early stage Crohn's disease}

$\mathrm{T}$ he presence of antibodies against the yeast Saccharomyces cerevisiae (ASCA) and against neutrophils (pANCA) has been used as diagnostic serological markers for inflammatory bowel disease (IBD) for many years. The combination of a positive ASCA test with a negative pANCA test has a positive predictive value of $96 \%$ and a specificity of $97 \%$ for Crohn's disease (CD). ${ }^{1}$ However, both antibodies have been found in other diseases, such as autoimmune liver disease, primary sclerosing cholangitis (pANCA), and in gluten sensitive enteropathy (ASCA). Therefore, their role as diagnostic serological markers for IBD seems to be limited.

Antibody determination is of interest in patients with indeterminate colitis. However, almost $50 \%$ of these patients do not develop ASCA or pANCA antibodies whereas in antibody positive patients, ASCA+/pANCA - predicts CD in $80 \%$ of patients with indeterminate colitis and ASCA-/pANCA+ predicts ulcerative colitis (UC) in $64 \%{ }^{2}$

Generation of both antibodies is poorly understood. Several studies have shown that titres of both antibodies do not correlate with disease activity, as known from classical autoimmune disease. Antibody titres seem to be stable over long periods of time. Surprisingly, pANCA in UC persist after colectomy, ${ }^{3}$ and we have observed patients who have had their last flare up of CD more than 20 years ago and currently display normal findings in gastroscopy, colonoscopy, and histology, but still have high titres of ASCA. Thus these antibodies seem to represent stable serological markers. The only clinical parameter confirmed by several groups is the correlation between ASCA positivity and ileal involvement of disease and penetration as well as structuring disease behaviour. ${ }^{4}$

The question has been raised whether pANCA and ASCA represent genetic markers for susceptibility to IBD. Several studies tried to elucidate this question. Family studies showed that $16-30 \%$ of healthy first degree relatives of patients with UC were pANCA positive. ${ }^{56}$ Although these studies could not be confirmed by others, probably due to that pANCA may be a genetic marker. ${ }^{7}$ Comparable with pANCA research, several studies showed that ASCA were detectable in $20-25 \%$ of first degree relatives of patients with $\mathrm{CD} .{ }^{8}{ }^{9}$ Healthy spouses however were generally antibody negative, indicating that genetic and not environmental factors play a decisive role. The prevalence of ASCA in families with more than two affected members suffering from $\mathrm{CD}$ was significantly higher than in families with only two affected members, which points towards the role of ASCA as a genetic marker. ${ }^{10}$ In the same study however, the prevalence of these serological markers did not differ in pure Crohn's families overall from sporadic cases. Therefore, the question needs to be raised whether these antibodies develop as an epiphenomenon during the onset of disease. It is known that luminal antigens such as bacteria and yeast seem to play an essential role for the perpetuation of inflammatory processes. In patients with CD, loss of immune tolerance towards the resident bacterial flora is one of the major pathogenetic concepts for this disease. Possibly, pANCA are due to cross reactivity to bacterial antigens. ${ }^{11}$ Bacterial and yeast antigens are ubiquitous, permanently present in the gastrointestinal tract. Therefore, it would be of great interest to evaluate when these antibodies are generated.

The study of Israeli and colleagues ${ }^{12}$ in this issue of Gut is the first to provide an answer to this question (see page 1232). In this study, ASCA were detected in $31 \%$ of patients before the clinical diagnosis of CD. Furthermore, an methodological problems, they indicate increase in ASCA frequency was observed over time, with the highest frequency documented in the 36 months before the diagnosis of $\mathrm{CD}$. These results indicate that ASCA development occurs before or during the early stages of disease. This thesis was confirmed by one patient who was ASCA negative 80 months before diagnosis but was ASCA positive 48 months before diagnosis. Hence ASCA do not seem to be generated as genetic markers in early childhood but in the context of early disease. ASCA may therefore be a marker of an immune response to an environmental antigen that occurs in the context of an early stage of disease. In some patients with other autoimmune diseases, such as lupus erythematosus and rheumatoid arthritis, antibodies were detected up to nine years before diagnosis. ${ }^{13}{ }^{14}$ However, whether inflammatory bowel disease (IBD) autoantibodies are markers of future disease, as has been suggested for classical autoimmune disease, has yet to be determined. If this hypothesis is true, the high frequency of ASCA in family studies would indicate that the frequency of diseased family members is substantially higher than actually known.

In Israeli's study, ${ }^{12}$ four of eight patients had an increase in ASCA titres whereas in two patients the titres decreased. The increase in titres was interpreted as crescendo autoimmunity by Israeli et al, although this finding must be interpreted carefully considering the small number of patients available. ${ }^{12}$ Furthermore, antibody titres in IBD are generally stable, in contrast with various other autoimmune diseases where there is a correlation between clinical activity and titre levels. Therefore, it is questionable whether IBD and other classical autoimmune diseases can be compared.

The initial event leading to IBD is still unclear but of major interest. ASCA positivity has been found to be associated with a deficiency in mannan binding lectin, a component of the innate immune system. ${ }^{15}$ The theory that the initial incident leading to IBD is an infection in patients with a defect in their innate immune system is still speculation. The data of Israeli et al can be interpreted in two ways: either the autoimmune reactions precede the disease or a latent subclinical disease is followed by generation of antibodies as an epiphenomenon. Which came first, the chicken or the egg?

Approximately seven decades after the first description of $\mathrm{CD}$, our knowledge about this disease is still limited. Above all we do not know the number of undiagnosed cases, if there are 
asymptomatic patients, or whether a subclinical form of CD exists, as is in the case of individuals who are hepatitis $\mathrm{C}$ virus RNA positive but have normal transaminases. Ten to 20 year since the description of ASCA and pANCA, the paper of Israeli and colleagues ${ }^{12}$ shows that ASCA and pANCA precede the clinical diagnosis of IBD. This study should encourage the scientific world to perform large studies where antibody positive and negative healthy family members are followed over a long period of time. This will help determine whether CD develops more frequently in antibody positive persons compared with those who are antibody negative. Furthermore, regular precise clinical observation may detect subclinical disease in antibody positive and negative patients.

Gut 2005;54:1212-1213.

doi: 10.1136/gut.2005.065227

Correspondence to: Professor F Seibold, Department of Gastroenterology, Inselspital, Freiburgstrasse, 3010 Bern, Switzerland frank.seibold@insel.ch
Conflict of interest: None declared.

\section{REFERENCES}

1 Quinton JF, Sendid B, Reumaux D, et al. AntiSaccharomyces cerevisiae mannan antibodies combined with antineutrophil cytoplasmic autoantibodies in inflammatory bowel disease: prevalence and diagnostic role. Gut 1998;42:788-91.

2 Joossens $S$, Reinisch W, Vermeire S, et al. The value of serologic markers in indeterminate colitis: a prospective follow-up study. Gastroenterology 2002; 122:1242-7.

3 Seibold F, Weber P, Klein R, et al. Clinical significance of antibodies against neutrophils in patients with inflammatory bowel disease and primary sclerosing cholangitis. Gut 1992;33:657-62.

4 Giaffer MH, Clark A, Holdsworth CD. Antibodies to Saccharomyces cerevisiae in patients with Crohn's disease and their possible pathogenic importance. Gut 1992;33:1071-5.

5 Shanahan F, Duerr R, Rotter J, et al. Neutrophil autoantibodies in ulcerative colitis: Familial aggregation and genetic heterogeneity. Gastroenterology 1992;103:456-61.

6 Seibold F, Slametschka D, Gregor M, et al. Neutrophil autoantibodies: a genetic marker in primary sclerosing cholangitis and ulcerative colitis. Gastroenterology 1994; 107:532-6.

7 Lee C, Lennard-Jones J, Cambridge G. Antineutrophil antibodies in familial inflammatory bowel disease. Gastroenterology 1995; 108:428-33.

8 Sendid B, Quinton J, Charrier G, et al. AntiSaccharomyces cerevisiae mannan antibodies in familial Crohn's disease. Am J Gastroenterol 1998;93:1306-10.

9 Seibold F, Stich O, Hufnagl R, et al. Anti-Saccharomyces cerevisiae antibodies in inflammatory bowel disease: a family study. Scand J Gastroenterol 2001;36:196-201.

10 Vermeire S, Peeters M, Vlietinck R, et al. AntiSaccharomyces cerevisiae antibodies (ASCA), phenotypes of IBD, and intestinal permeability: a study in IBD families. Inflamm Bowel Dis 2001;7:8-15.

11 Seibold F, Brandwein S, Simpson S, et al. pANCA represent a crossreactivity to enteric bacterial antigens. J Clin Immunol 1998; 18:153-60.

12 Israeli E, Grotto I, Gilburd B, et al. AntiSaccharomyces cerevisiae and antineutrophil cytoplasmic antibodies as predictors of inflammatory bowel disease. Gut 2005;54:1232-6.

13 Arbuckle MR, McClain MT, Rubertone MV, et al. Development of autoantibodies before the clinical onset of systemic lupus erythematosus. N Engl J Med 2003;349:1526-33.

14 Scofield RH. Autoantibodies as predictors of disease. Lancet 2004;363:1544-6.

15 Seibold F, Konrad A, Flogerzi B, et al. Genetic variants of the MBL are associated with immune reactivity to Saccharomyces cerevisiae in Crohn's disease. Gastroenterology 2004; 127:1076-84.
IFN- $\gamma$ itself and is capable of promoting IFN- $\gamma$ production, not only in Thl cells, but also in Th2 cells. T-bet also increases expression of the IL-12Rß2 chain. Macrophage and dendritic cell derived IL-12 is crucial in Thl immune responses. ${ }^{5}$ The IL- 12 receptor is made of two chains, $\beta 1$ and $\beta 2$, but only the $\beta 2$ chain can signal and phosphorylate STAT4 which then migrates to the nucleus and further boosts IFN- $\gamma$ production. Other factors also augment Thl polarisation. IL-18 activates the transcription factors AP-1 and nuclear factor $\kappa \mathrm{B}$ in $\mathrm{T}$ cells, and acts synergistically with IL-12 to boost IFN production. Type 1 interferons can also activate STAT4. IL-7, IL-15, and IL-21 also act in synergy with IL-12 to boost IFN- $\gamma$ production and all, including IL-18, are overexpressed in Crohn's disease. ${ }^{6}$ The IL-12 p40 chain can form a heterodimer with p19 protein to form a recently described cytokine, IL-23, which appears to be important in the activation of memory Thl cells. ${ }^{5}$ There are no publications on IL-23 in Crohn's disease.

In this issue of Gut, Sato and colleagues $^{7}$ identify osteopontin (also known as early T lymphocyte activation Eta-1) as yet another cytokine involved in the Thl response of Crohn's disease (see page 1254). Osteopontin is a $60 \mathrm{kDa}$ phosphoprotein constitutively secreted by epithelial cells and bone. ${ }^{8}$ It contains the characteristic RGD sequence seen in extracellular matrix proteins and shares receptor binding on cells with of the interleukin 12 (IL-12) receptor, and activated STAT4, all indicate that the mucosal environment in Crohn's disease favours Thl polarisation. ${ }^{1}$
Factors which commit virgin $\mathrm{T}$ cells to the Th1 or Th2 pathway are still under investigation. T-bet appears to be of primary importance. ${ }^{4}$ It is induced by 


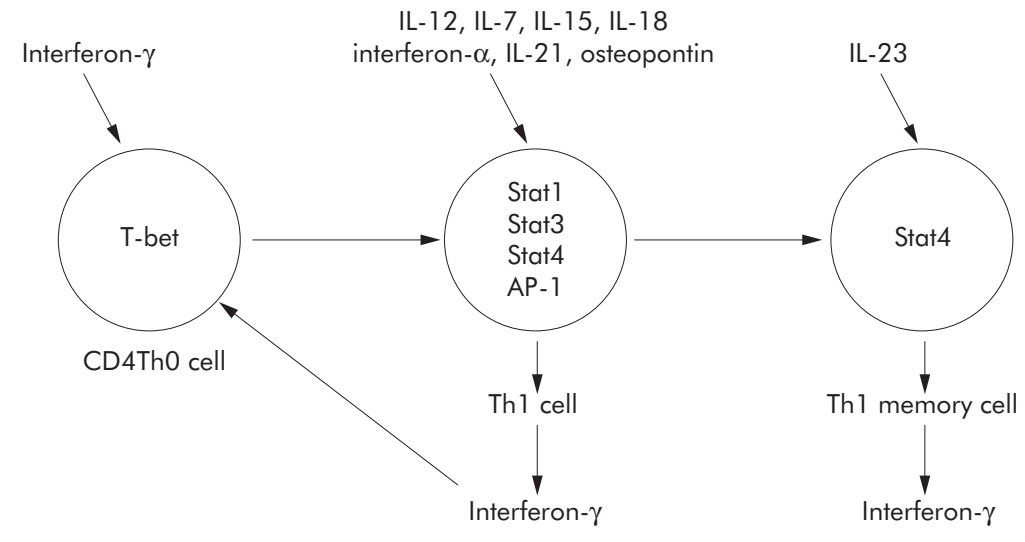

Figure 1 Diagrammatic indication of the cytokines, transcription factors, and signalling molecules which polarise virgin CD4 cells into Thelper cell type I cells. Interferon $\gamma$ (IFN- $\gamma$ ) induces the transcription factor T-bet which induces the high affinity interleukin (IL)- 12 receptor on T cells. IL-12 can then bind to its receptor, and activate STAT4, which goes to the nucleus and activates IFN- $\gamma$ transcription. A plethora of other cytokines can synergise with IL-12 to boost IFN- $\gamma$ production. IL-23, an IL-12-like cytokine, seems to be more important in activating memory cells. A crucial component of this pathway is the feedback loop by which IFN- $\gamma$ continues to drive T cells along a Th1 pathway.

extracellular matrix proteins, including $\alpha \mathrm{v}$ and $\beta 1$ integrins. It also binds to CD44. Osteopontin is expressed in many distinct forms depending on differential splicing and the glycosylation/phosphorylation status of the core protein. Proteolytic cleavage by thrombin and matrix metalloproteinases also changes its function. Osteopontin is important in many different tissues. In the bone it regulates calcium deposition and it is also a chemoattractant for cancer cells. However, it is also important in the immune system as it is made by activated $\mathrm{T}$ cells, macrophages, and dendritic cells. These cells also have osteopontin receptors. ${ }^{9}$

Osteopontin increases the adhesion of activated $\mathrm{T}$ cells and is a T cell chemoattractant, but importantly it supports Thl responses and inhibits Th2 responses; thus its identification in Crohn's disease adds another member to the increasing number of cytokines which drive Thl immune responses (fig 1). Osteopontin deficient mice show impaired Thl immune responses and fail to make granulomas.' There was therefore a strong rationale for looking at osteopontin in Crohn's disease. Sato and colleagues $^{7}$ now show that osteopontin transcripts and protein are elevated in Crohn's disease and ulcerative colitis. Osteopontin also increases IL-12 production specifically in Crohn's disease lamina propria mononuclear cells but has little effect on IL-10 production. Arguably, the most important finding of the paper is the source of the osteopontin. There was a predictable increase in immunoreactivity in epithelial cells, macrophages, and around granulomas in Crohn's disease. However, the most striking feature in inflammatory bowel disease (IBD) was coexpression of osteopontin with mucosal IgM, IgG, and IgA plasma cells. Especially in Crohn's disease, osteopontin was particularly associated with IgG plasma cells. This result has some similarities with a previous study on osteopontin in Crohn's disease and normal ileum where production in plasma cells was also noted, although overall no differences were seen between controls and Crohn's samples. ${ }^{10}$ However, gene array analysis has shown markedly increased osteopontin transcripts in ulcerative colitis colon compared with normal colon. ${ }^{11}$

The role of plasma cells in IBD has been somewhat overlooked in recent years, with most emphasis on cellular immunity. However, quantitatively, plasma cells are as abundant as T cells in normal and inflamed gut. By far the largest population of plasma cells in normal and inflamed gut secrete IgA, generally considered to be a beneficial non-phlogistic antibody. In IBD, the largest quantitative increase is in $\operatorname{IgA}$ plasma cells, but the biggest proportional increase is in IgG plasma cells as these are uncommon in the normal gut. In Crohn's disease, IgG plasma cells tend to be found around ulcers whereas in ulcerative colitis they are present along the length of the diseased mucosa. ${ }^{12}$ It has been suggested that some of this IgG has specificity for gut autoantigens, such as epithelial tropomysin, indicating that at least part of the pathogenesis of ulcerative colitis may involve antibody mediated autoimmunity. ${ }^{12}$ The results of Sato et al suggest that IgA and IgG plasma cells may be far more important than previously considered, with a role greater than simply being antibody secreting factories.

The work of Sato and colleagues ${ }^{7}$ also poses some interesting questions. Is osteopontin always made by plasma cells and is its presence in the gut merely a feature of the fact that the gut contains more plasma cells than the rest of the body combined? Do plasma cells secrete bioactive osteopontin? If osteopontin is present in plasma cells in healthy gut, why does it not deliver a survival signal to CD4 cells? Is osteopontin involved in the migration of $\mathrm{T}$ cells into normal and inflamed gut? It would also be of interest to examine trinitrobenzene sulphonic acid colitis in osteopontin deficient mice.

The importance of the therapeutic benefit of neutralising Thl inducing cytokines in Crohn's disease is well demonstrated by the clinical success of anti-IL-12 antibodies. ${ }^{13}$ However, the antibody used in this study is against the p40 subunit of IL-12 and theoretically can also neutralise IL-23. However, there may be considerable heterogeneity between patients in the relative importance of other Thl inducing cytokines, such as IL-18 and osteopontin, which deserve further investigation.

Gut 2005;54:1213-1215.

doi: $10.1136 /$ gut.2004.063412

..................

Authors' affiliations

J N Gordon, T T MacDonald, Division of Infection, Inflammation, and Repair, University of Southampton School of Medicine,

Southampton General Hospital, Southampton, UK

Correspondence to: Professor T T MacDonald, Bart's and the London School of Medicine and Dentistry, Turner St, London El 2AD, UK; t.t.macdonald@soton.ac.uk

Conflict of interest: None declared.

\section{REFERENCES}

1 MacDonald TT, Monteleone G. Immunity, inflammation and allergy in the gut. Science 2005;307:1920-25.

2 MacDonald TT, Monteleone G. IL-12 and Th 1 immune responses in human Peyer's patches. Trends Immunol $2001 ; 22: 244-7$.

3 Sturm A, Leite AZ, Danese S, et al. Divergent cell cycle kinetics underlie the distinct functional capacity of mucosal T cells in Crohn's disease and ulcerative colitis. Gut 2004;53:1624-31.

4 Weigmann B, Neurath MF. T-bet and mucosal Thl responses in the gastrointestinal tract. Gut 2002;51:301-3.

5 Trinchieri G, Pflanz S, Kastelein RA. The IL-12 family of heterodimeric cytokines: new players in the regulation of T cell responses. Immunity 2003;19:641-4.

6 MacDonald TT, Monteleone G. Role of the immune system in the pathogenesis of IBD. In Immune mechanisms in IBD. In: Neurath MF, Blumberg R, eds. New York: Landes Bioscience (in press).

7 Sato T, Nakai T, Tamura N, et al. Osteopontin/ Eta-1 upregulated in Crohn's disease regulates 
the Th1 immune response. Gut 2005; 54: 1254-62.

8 Sodek J, Zhu B, Huynh MH, et al. Novel functions of the matricellular proteins osteopontin and osteonectin/SPARC. Connect Tissue Res 2002;43:308-19.

9 O'Regan AW, Nau GJ, Chupp GL, et al. Osteopontin (Eta-1) in cell-mediated immunity: teaching an old dog new tricks. Immunol Today 2000;21:475-8.

10 Gassler N, Autschbach F, Gauer S, et al. Expression of osteopontin (Eta-1) in Crohn disease of the terminal ileum. Scand J Gastroenterol 2002;37:1286-95.

11 Masuda H, Takahashi Y, Asai S, et al.

Distinct gene expression of osteopontin in patients with ulcerative colitis. J Surg Res 2003;111:85-90.

12 Brandtzaeg P. The B cell system. In: Brostoff J Challacombe SJ, eds. Food allergy and intolerance. London: Bailliere Tindall, 1987:118-55.

13 Mannon PJ, Fuss IJ, Mayer L, et al. Anti-

interleukin-12 antibody for active Crohn's disease. N Engl J Med 2004;351:2069-79.

\section{Silencing RNA: a novel treatment for pancreatic cancer?}

\section{N R Lemoine}

\section{The high sequence specificity of RNA interference may make it suitable to treat diseases that are linked to selective or elevated expression of particular identified genes, such as in pancreatic cancer}

$\mathrm{T}$ he antiapoptotic gene Bcl-2 has been a target for downregulation by nucleic acid based strategies for more than a decade but the recent failure of the synthetic antisense oligonucleotide agent Genasense in phase III clinical trial caused many to think again about the worth of this approach. However, a new optimism about this and other targets is spreading through the community following several encouraging applications of the recently discovered technology of RNA interference, which is essentially a new biological version of the antisense system. ${ }^{1}$

RNA interference is considered to have begun as an evolutionarily ancient mechanism for protecting organisms from viruses. Many viruses have RNA, rather than DNA, as their genetic material and go through at least one stage in their life cycle in which they make double stranded RNA. Perhaps not surprisingly, all multicellular organisms have evolved a well conserved protein apparatus that destroys double stranded RNA but this has also been found to play a role in maintenance of the organism's own genome stability by suppressing the movement of mobile genetic elements, such as transposons and repetitive sequences.

The gene silencing process of RNA interference (RNAi) involves the manufacture of short double stranded RNA molecules by an enzyme called DICER, which cleaves RNA duplexes into 21-26 base pair oligomers. These small interfering RNAs (siRNA) cause sequence specific, post-transcriptional gene silencing by guiding an endonuclease, the RNAi induced silencing complex (RISC), to mRNA. This process has been seen in a wide range of organisms such as Neurospora fungus (in which it is known as quelling), plants (post-transcriptional gene silencing), and mammalian cells (RNAi). Downregulation of target gene expression has been found to involve interactions at multiple levels. Where there is complete or near complete sequence complementarity between the small RNA and the target, the Argonaute 2 component of RISC mediates cleavage of the target transcript. ${ }^{2}$ In contrast, where there is sequence mismatch between the miRNA and the target transcript, the mechanism appears to involve repression of translation predominantly. ${ }^{4}$ More recently, it has been recognised that siRNA molecules can induce transcriptional silencing through promoter methylation. ${ }^{56}$

In principle, the high sequence specificity of RNA interference might make it suitable to treat disease that is linked to selective or elevated expression of particular identified genes. This may make it particularly appropriate for combating cancers associated with mutated endogenous gene sequences. An early example of the potential power of this approach came in a study of pancreatic cancer. RAS genes are frequently mutated in human cancers, particularly in pancreatic and colon carcinomas. Mutant RAS oncogenes often contain point mutations that alter only a single amino acid, which locks the oncogenic RAS proteins in a persistently activated
GTP bound state. A complication in using RAS oncogenes as targets in anticancer therapy is that at present it is not possible specifically to inhibit the biochemical function of only the oncogenic RAS alleles. This may be essential as the wild-type $K$-RAS gene appears to be required for viability, as evidenced by the embryonic lethal phenotype of mice nullizygous for $K$-ras. ${ }^{7}$ Retroviral delivery of siRNAs can specifically inhibit the mutant K-RASV12 allele in human pancreatic carcinoma cells, while leaving the wild-type $K$-RAS allele untouched. ${ }^{8}$ In spite of the fact that pancreatic carcinoma cells have many genetic alterations, loss of K-RASV12 expression leads to loss of tumorigenicity in experimental animal models.

In this issue of Gut, Ocker and colleagues $^{9}$ explore the use of siRNAs against another gene that is aberrantly expressed at high frequency in pancreatic cancer, the antiapoptotic gene bcl-2 (see page 1298). Their results suggest that the target can be selectively downregulated in tumour cells in vitro and that intraperitoneal administration of the naked nucleic acid agent can produce variable antitumour effects against malignant deposits growing subcutaneously in vivo. In this study-as in most experiments using RNAi to target particular genes in mammalian cellsthe results are interpreted as representing induction of sequence specific transcript cleavage. However, at this early stage in our understanding of RNAi, it is important not to rule out the possibility that interference mediated through protein translational repression or genomic modification (DNA methylation or histone modification) may also be playing a role in mediating gene specific silencing and any derived RNAi phenotype. The great attraction of therapeutic epigenetic gene specific silencing lies in its heritable nature, meaning that, unlike post-transcriptional gene silencing that requires the continued presence of an siRNA molecule targeting a coding sequence, long lasting suppression of gene expression could be achieved from a single exposure to a specific methylation inducing RNAi agent targeting a promoter sequence.

The major challenge in turning RNA interference into an effective therapeutic strategy is the delivery of RNA 
interference agents, whether they are synthetic, short double stranded RNAs (as in the paper by Ocker and colleagues $^{9}$ ) or viral vectors directing production of double stranded RNA, to the target cells within the body. While siRNA technology has proven extremely powerful and robust for cell culture work, translating this success reliably to animals or humans is proving very difficult, due to insufficient bioavailability of the compounds. However, an important step in the right direction is that Jürgen Soutschek and colleagues have recently been able to demonstrate siRNA mediated downregulation of apolipoprotein B in the liver (and jejunum) of mice using cholesterol conjugates delivered systemically. ${ }^{10}$ The effects were preferentially seen in the liver, which is a relatively easy organ to target, and relatively high dosages (three injections each of $50 \mathrm{mg} / \mathrm{kg}$ ) were required for the effect. In view of the extremely high potency of the siRNA in in vitro cell cultures, one must conclude that only a very small fraction of the injected siRNA actually reaches its molecular mRNA target in liver cells. Thus it is unfortunately not likely that simple cholesterol conjugation will solve the general delivery problem of siRNA. Other cationic cell penetrating peptides such as penetratin, Tat, and more recently transportan and oligo arginine have been proposed as general transmembrane carriers for a variety of cargoes, including oligonucleotides and PNA. ${ }^{11}{ }^{12}$ However, it appears that the main uptake route for most, if not all, of these peptides is endosomal, and thus the reagents have to escape the endosomal compartment in order to enter the cellular compartments of action: the cytoplasm and/or nucleus. The problem of cellular delivery is yet more complex for clinical application where the real challenge for an adjuvant therapy agent is delivery and maintenance of the compound in cancer cells in multiple organs in humans. However, the biotechnology industry has readily recognised the potential silencing properties of RNA mediated interference, with at least 15 companies active in the field, and two companies (Ribopharma and Benitec) have patents for RNAi based clinical applications. Significant progress in delivery technology is required before the concept of RNAi can realistically benefit cancer patients, but exploitation of the decade of clinical experience of antisense and viral gene therapy agents gives investigators a head start.

Gut 2005;54:1215-1216.

doi: $10.1136 /$ gut.2005.067108

Correspondence to: Professor $\mathrm{N}$ Lemoine, Cancer Research UK Molecular Oncology Unit, Barts and the London School of Medicine and Dentistry, Queen Mary University of London, Charterhouse Square, London ECI 6BQ, UK' nick.lemoine@cancer.org.uk
Conflict of interest: None declared.

\section{REFERENCES}

1 Hannon GJ. RNA interference. Nature 2002;418:244-51.

2 Liu J, Carmell MA, Rivas FV, et al. Argonaute2 is the catalytic engine of mammalian RNAi. Science 2004:305:1437-41.

3 Meister G, Landthaler M, Patkaniowska A, et al. Human Argonaute2 mediates RNA cleavage targeted by miRNAs and siRNAs. Mol Cell 2004; 15:185-97.

4 Kim J, Krichevsky A, Grad Y, et al. Identification of many microRNAs that copurify with polyribosomes in mammalian neurons. Proc Natl Acad Sci U S A 2004; 101:360-5.

5 Kawasaki H, Taira K. Induction of DNA methylation and gene silencing by short interfering RNAs in human cells. Nature 2004;431:21 1-17.

6 Morris KV, Chan SW, Jacobsen SE, et al. Small interfering RNA-induced transcriptional gene silencing in human cells. Science 2004;305: 1289-92

7 Johnson L, Greenbaum D, Cichowski K, et al. $\mathrm{K}$-ras is an essential gene in the mouse with partial functional overlap with N-ras. Genes Dev 1997;11:2468-81.

8 Brummelkamp TR, Bernards R, Agami R. Stable suppression of tumorigenicity by virus-mediated RNA interference. Cancer Cell 2002;2:243-7.

9 Ocker M, Neureiter D, Lueders M, et al. Variants of bcl-2 specific siRNA for silencing antiapoptotic $\mathrm{bcl}-2$ in pancreatic cancer. Gut 2005; 54:1298-1308

10 Soutschek J, Akinc A, Bramlage B, et al. Therapeutic silencing of an endogenous gene by systemic administration of modified siRNAs. systemic administration of
Nature 2004;432:173-8.

11 Jarver P, Langel U. The use of cell-penetrating peptides as a tool for gene regulation. Drug Discov Today 2004;9:395-402.

12 Wender PA, Jessop TC, Pattabiraman K, et al. An efficient, scalable synthesis of the molecular transporter octaarginine via a segment doubling strategy. Org Lett 2001;3:3229-32.

\section{Sir Francis Avery Jones BSG Research Award 2005}

Applications are invited by the Education Committee of the British Society of Gastroenterology who will recommend to Council the recipient of the 2006 Award. Applications (20 copies) should include:

- A manuscript (2 A4 pages only) describing work conducted

- A bibliography of relevant personal publications

- An outline of the proposed content of the lecture, including title

- A written statement confirming that all or a substantial part of the work has been personally conducted in the UK or Eire.

Entrants must be 40 years or less on 31 December 2005 but need not be a member of the Society. The recipient will be required to deliver a 30 minute lecture at the annual meeting of the Society in Birmingham in March 2006. Applications (20 copies) should be made to the Honorary Secretary, British Society of Gastroenterology, 3 St Andrews Place, London, NW1 4LB by 1 December 2005. 